Indonesian Journal of Biotechnology, June, 2013

Vol. 18, No. 1, pp.14-25

\title{
Phylogeny and Origin of Glucose-6-Phosphate Dehydrogenase (G6PD) Deficiency Mutations in Indonesia
}

\author{
Maria Omega ${ }^{1}$ and Ross T. Barnard ${ }^{1^{*}}$
}

${ }^{1}$ Biotechnology Program, School of Chemistry and Molecular Biosciences, The University of Queensland, Australia

\begin{abstract}
The aim of this study is to analyze the relationship between the types of G6PD mutations found in Indonesia and the relationships of mutations found in Indonesia to those found in other countries. We summarize the distribution of G6PDs in West Indonesia and East Indonesia. Moreover, we use bioinformatics methods to construct phylogenetic trees and compare the sequences containing the regions amplified by the commonly used PCR primer pairs. Previous work has shown that Mediterranean G6PD and Chinese CoimbraG6PD are distributed in West Indonesia, whilst G6PD mutations in East Indonesia are Jammu/ViangchanG6PD and Chinese Gaohe G6PD. G6PD Jammu/Viangchan was mostly distributed in Flores Island, East Indonesia along with G6PDGaohe. We constructed phylogenetic trees using the G6PD sequences from various regions in Indonesia and other countries. It appears from phylogenetic trees and percentages of identity that Flores Indonesian G6PD deficiency (Jammu/Viangchan G6PD, originating in India) is 92.5\% identical to the G6PD deficiency of Chinese origin (GaoheG6PD). It was interesting to note that the genetic region containing the Javanese Indonesian G6PD deficiency (MediterraneanG6PD, first found in Italy) located in the western parts of Indonesia is closely related (99\% identity) to the Chinese G6PD deficiency(Coimbra G6PD). We conclude that G6PD mutations in West Indonesia are closely related to G6PD mutations from China. G6PD mutations in East Indonesia are also closely related to G6PD mutations from India and China, but more distantly, and to different types to those in West Indonesia. A prediction of protein structure was carried out which allowed visualization of the locations of mutation on the three dimensional structure of G6PD.
\end{abstract}

Key words: G6PD, phylogeny, origin, genetic mutations

\section{Introduction}

Glucose-6-phosphate dehydrogenase (G6PD) deficiency is a metabolic enzyme involved in the pentose phosphate pathway, particularly vital in red blood cell metabolism. Frank (2005) found thatG6PD deficiency is the most common human enzyme defect, which is an X-linked recessivehereditary disease characterized by abnormally low levels of glucose-6-phosphate dehydrogenase. Moreover, G6PD Deficiency is now known to be slightly more common than previously

*Corresponding author:

Ross T. Barnard

The University of Queensland, Brisbane QLD 4072,

Australia phone: +61-07-33654612

email: rossbarnard@uq.edu.au thought in women though the symptoms are generally not as severe unless both $X$-chromosomes are affected. When it was discovered that G6PD Deficiency was a genetic disorder by Beutler et al. (1991) it wasthought to be a recessive disorder. Since women have two copies of the $\mathrm{X}$-chromosome, they believed women would not be affected unless both chromosomes had the G6PD Deficiency mutation. The current view is that symptomatic patients are almost exclusively male, due to the $X$-linked pattern of inheritance, but female carriers can be clinically affected due to unfavorable lyonization, where random inactivation of an X-chromosome in certain cells creates a population of G6PD-deficient red blood cells 
coexisting with normal red cells (Hamilton et al., 2004)

Mazza (2001) revealed that there are 440 different variants and the G6PD classification is divided by five types:

1. Chronic haemolytic anemia ( $<10 \%$ activity with severe deficiency).

2. Intermittent haemolysis ( $<10 \%$ activity with severe deficiency).

3. Haemolysis with stressors (10-60\% activity with mild deficiency).

4. No clinical sequelae (non-deficient variant).

5. No clinical sequelae (increased enzyme activity).

Cappellini and Fiorelli (2008) showed that G6PD is the most common human enzyme defect, being present in more than 400 - 600 million people worldwide $(8.96 \%$ of the world population). African, Middle Eastern/Mediterranean, European and South East Asian people are affected the most, along with those who are mixed with any of the above. Mehta et al. (2000) studied that a side effect of this disease is that it confers protection against malariain particular the form of malaria caused by Plasmodium falciparum, the most deadly form of malaria. A similar relationship exists between malaria and sickle-cell disease. This is because the cells infected with the Plasmodium parasite are cleared more rapidly by the spleen (Monga et al.,2003). This phenomenon might give G6PDH deficiency carriers an evolutionary advantage by increasing their fitness in malarial endemic environments. Specifically, Indonesia carries one of the highest burdens of malaria in the world, and Primaquine (PQ) as the only available drug for radical cure of Plasmodium vivax and prevention of $P$. falciparum transmission. The PQ can trigger acute haemolysis in G6PD deficient individuals and cause problems in malaria treatment. Furthermore, Satyagraha (2012) reported thatin Indonesia, G6PD rates are relatively high in the many different and geographically isolated ethnic groups. The background of the study is that G6PD 1388 G>A (known as Kaiping), which is a variant suggested to be of Chinese origin, is found in Southeast Asia. G6PD Kaiping is widely distributed among native Flores and Palue Islanders for unknown reasons, while G6PD Canton (1376 G>T) is very rare or entirely absent in these locations (Tantular et al., 2010). Therefore, it is interesting to investigate whether these variants or other found in Indonesia arose by local mutations or were introduced by Chinese or other migrants. To elucidate the answers, bioinformatics analysis is needed, such as a haplotype study of Xq28 of Chinese, Indian and Indonesian people. In the present study, we used bioinformatics to investigate the relationships between the mutations found in Indonesia and those found in other countries.

\section{Materials and Methods}

Sequence data was compared to the GenBank DNA Database using BLASTn searches to determine the alignment (\% identity) between primers and sequences containing G6PD mutations using the National Centre for Biotechnology Information (NCBI) BLAST network server available from http:/ / www.ncbi.nlm.nih.gov/

\section{Construction of phylogenetic trees.}

The materials are the computers and the software such as ClustalX v 2.1 to construct phylogenetic trees. The phylogenetic trees were developed to test the relationship between regions of sequence containing the squences bounded by PCR primers which were aligned against a selection of genbank database sequences using ClustalX v 2.1 available from http://www.clustal.org/.We used G6PD mutations that cause G6PD deficiency. They are located on the long arm of $X$ chromosome, on band Xq28 (McKusick and Hamosh, 2000). The G6PD gene spans 18.5 kilobases (Warrel et al., 2005). Sequences based on databases were uploaded as FASTA files and a preliminary complete alignment was done. Regions containing sequences defined by primers 
were aligned to draw bootstrapped NJ trees (using 1000 bootstrap steps) and the trees were visualized using Treeview.

\section{Results}

Sets of primers to identify the G6PD deficiency and mutations

There are 14 sets of primers that have been used previously to identify the G6PD deficiency in Indonesian, Chinese and Indian people (Table 1, 2 and 3).

There are 13 G6PD sequences from NCBI used in this report (Table 4).
Mutations of G6PD deficiency

G6PD protein was submitted to http:/ / zhanglab.ccmb.med.umich.edu/I-TASSER/ output/S110585

\section{Phylogenetic trees}

Phylogenetic tree (Figure 3) was generated based on a block of $910 \mathrm{bp}$ that includes mutations at 1376 and at 392 in adjacent regions 3 and 4 of G6DP on chromosome Xq28 (Table 8).

Table 1. Different Sets of G6PD primers used in Indonesian people

\begin{tabular}{|c|c|c|c|c|c|}
\hline $\begin{array}{l}\text { G6PD } \\
\text { mutation }\end{array}$ & $\begin{array}{c}\text { Sense/ } \\
\text { antisense } \\
\text { strand } \\
\end{array}$ & G6PD primers (nucleotides) & $\begin{array}{c}\text { Restriction } \\
\text { Enzymes }\end{array}$ & $\begin{array}{l}\text { Design } \\
\text { Locations }\end{array}$ & References \\
\hline $\mathrm{nt1376}\left(\mathrm{G}^{\rightarrow} \mathrm{T}\right)$ & $\begin{array}{l}\text { Sense } \\
\text { Antisense }\end{array}$ & $\begin{array}{l}\text { 5'-ACGTGAAGCTCCTGACGC-3' } \\
5^{\prime} \text {-TGAAAATACGCCAGGCCTCG } \\
-3^{\prime}\end{array}$ & XhoI & $\begin{array}{l}\text { Indonesia } \\
\text { China }\end{array}$ & $\begin{array}{l}\text { Matsuoka et al. (2003) } \\
\text { Kawamoto et al. (2006) } \\
\text { Soemantri et al. (1995) }\end{array}$ \\
\hline $\mathrm{nt1388}(\mathrm{G} \rightarrow \mathrm{A})$ & $\begin{array}{l}\text { Sense } \\
\text { Antisense }\end{array}$ & $\begin{array}{l}\text { 5'-ACGTGAAGCTCCTGACGC-3' } \\
\text { 5'-GTGCAGCAGTGGGGTGAA } \\
\text { CATA-3' }\end{array}$ & NdeI & $\begin{array}{l}\text { Indonesia } \\
\text { China }\end{array}$ & $\begin{array}{l}\text { Matsuoka et al., (2003) } \\
\text { Kawamoto et al., (2006) } \\
\text { Soemantri et al., (1995) } \\
\text { Tantular et al., (2010) }\end{array}$ \\
\hline nt95 $\left(\mathrm{A}^{\rightarrow} \mathrm{G}\right)$ & $\begin{array}{l}\text { Sense } \\
\text { Antisense }\end{array}$ & $\begin{array}{l}5 \\
\text { CGTTCACAAGGAGTGATTTG-3' } \\
\text { 5' -CGATGCACCCATGATGAT } \\
\text { GAATACG-3' }\end{array}$ & MluI & $\begin{array}{l}\text { Indonesia } \\
\text { China }\end{array}$ & $\begin{array}{l}\text { Matsuoka et al. (2007), } \\
\text { Tantular et al. (2010) }\end{array}$ \\
\hline $\mathrm{nt1024}\left(\mathrm{C}^{\rightarrow \mathrm{T}}\right)$ & $\begin{array}{l}\text { Sense } \\
\text { Antisense }\end{array}$ & $\begin{array}{l}\text { 5'-GTCAAGGTGTTGAAATGCA } \\
\text { TC-3' } \\
\text { 5'-CATCCСАССТСТCATTCTT } \\
\text { CС-3' }\end{array}$ & MboII & $\begin{array}{l}\text { Indonesia } \\
\text { China }\end{array}$ & $\begin{array}{l}\text { Matsuoka et al. (2003), } \\
\text { Kawamoto et al. (2006) }\end{array}$ \\
\hline $\mathrm{nt} 487\left(\mathrm{C}^{\rightarrow \mathrm{T}}\right)$ & $\begin{array}{l}\text { Sense } \\
\text { Antisense }\end{array}$ & $\begin{array}{l}\text { 5'-GCGTCTGAATGATGCTGCT } \\
\text { GTGAT-3' } \\
\text { 5'-AGCCGGTCAGTGCTCTGC } \\
\text { ATGTCC-3' }\end{array}$ & AluI & $\begin{array}{l}\text { Indonesia } \\
\text { India }\end{array}$ & $\begin{array}{l}\text { Matsuoka et al. (2003), } \\
\text { Kawamoto et al. (2006) }\end{array}$ \\
\hline
\end{tabular}

Table 2. Different Sets of G6PD primers used in Chinese people (Matsuoka et al., 2007)

\begin{tabular}{|c|c|c|c|}
\hline G6PD mutation & Sense/antisense & G6PD Primers & $\begin{array}{c}\text { Nucleotide size in } \\
\text { basepairs (bp) }\end{array}$ \\
\hline \multirow{2}{*}{$\mathrm{nt175}\left(\mathrm{C}^{\rightarrow} \mathrm{T}\right)$} & Sense & 5'-ACATGTGGCCCCTGCACCACA-3' & $242 \mathrm{bp}$ \\
\hline & Antisense & 5'-GTGACTGCTCTGCCACCCTG-3' & \\
\hline \multirow{2}{*}{$\mathrm{nt163}\left(\mathrm{C}^{\rightarrow} \mathrm{T}\right)$} & Sense & 5'-TTGGGGTCCCCATGCCCTTG-3 & $231 \mathrm{bp}$ \\
\hline & Antisense & 5'-TGCCTCGTCACAGATGGGCC-3' & \\
\hline \multirow{2}{*}{$\mathrm{nt1376}(\mathrm{G} \rightarrow \mathrm{T})$} & Sense & 5'-CTGAGAGAGCTGGTGCT-3' & $342 \mathrm{bp}$ \\
\hline & Antisense & 5'-CACCATGTGGAGTCCCCCGG-3' & \\
\hline \multirow{2}{*}{$\operatorname{nt} 1311\left(C^{\rightarrow T}\right)$} & Sense & 5'-ACTTCCACATGGTGGCAGGCAG-3' & $397 \mathrm{bp}$ \\
\hline & Antisense & 5'-ATGAGGTAGCTCCACCCTCA-3' & \\
\hline \multirow{2}{*}{$\mathrm{nt} 1388(\mathrm{G} \rightarrow \mathrm{A})$} & Sense & 5'-TGAGGGTGGAGCTACCTCAT-3' & $164 \mathrm{bp}$ \\
\hline & Antisense & 5'-CGGGGGTGGAGGTGGGTGCCCA-3' & \\
\hline
\end{tabular}


Table 3. Different Sets of G6PD primers used in Indian people (Vulliamy et al., 1991)

\begin{tabular}{|c|c|c|c|}
\hline G6PD mutation & Sense/antisense & G6PD Primers & Restriction enzyme \\
\hline \multirow{2}{*}{$n+175\left(C^{\rightarrow T}\right)$} & Sense & 5'-TGGACCCCTACACAGCCAAGTAC-3' & ScaI and SacI \\
\hline & Antisense & 5'-GGCATGCTCCTGGGGACTGCT-3' & \\
\hline \multirow{2}{*}{$\mathrm{nt} 163\left(\mathrm{C}^{\rightarrow} \mathrm{T}\right)$} & Sense & 5'-GGAGCTAAGGCGAGCTCTGGC-3' & BspHI \\
\hline & Antisense & 5'-TGCCTTGCTGGGCCTCGAAGG-3' & \\
\hline \multirow{2}{*}{$\mathrm{nt563}\left(\mathrm{C}^{\rightarrow} \mathrm{T}\right)$} & Sense & 5'-ACTCCCGAAGAGGGGTTCAAGG-3' & MboII \\
\hline & Antisense & 5'-CCAGCСТCСCAGGAGAGAGGAAG-3' & \\
\hline \multirow[t]{2}{*}{$\mathrm{nt} 1311\left(\mathrm{C}^{\rightarrow} \mathrm{T}\right)$} & Sense & $5^{\prime}$-TGTTCTTCAACCCCGAGGAGT-3' & BclI \\
\hline & Antisense & 5'-AAGACGTCCAGGATGAGGTGATC-3' & \\
\hline
\end{tabular}

Table 4. G6PD sequences, their origins and distributions

\begin{tabular}{|c|c|c|c|}
\hline No & G6PD and its sequences in NCBI & Distributions & $\begin{array}{l}\text { G6PD mutation } \\
\text { and names }\end{array}$ \\
\hline 1. & $\begin{array}{l}\text { >gi|108773792 | ref | NM_001042351.1| Homo sapiens } \\
\text { glucose-6-phosphate dehydrogenase (G6PD), transcript } \\
\text { variant 2, exon 11, mRNA } \\
\text { Origins: Kobe, Japan; Telti, Italy (Mason et al., 1995) }\end{array}$ & $\begin{array}{l}\text { Israel, Jordan, Cairo, } \\
\text { Mediterranean, } \\
\text { Uzbekistan, India, China }\end{array}$ & $\begin{array}{l}\text { nt1318 }(\mathrm{C}>\mathrm{T}) \\
\text { Telti }\end{array}$ \\
\hline 2. & $\begin{array}{l}\text { >gi }|108773794| \text { ref } \mid \text { NM_000402.3 |Homo sapiens glucose- } \\
\text { 6-phosphate dehydrogenase (G6PD), transcript variant 1, } \\
\text { exon 11, mRNA } \\
\text { Origins: Britain, UK; Iran (Mortazavi et al., 1997) }\end{array}$ & $\begin{array}{l}\text { Israel,Jordan, Cairo, } \\
\text { Mediterranean, } \\
\text { Uzbekistan, India, China }\end{array}$ & $\begin{array}{l}\text { nt1311 }(\mathrm{C}>\mathrm{T}) \\
\text { Iranian/European }\end{array}$ \\
\hline 3. & $\begin{array}{l}\text { >gi }|213385264| \text { ref }\left|N G \_009015.1\right| \text { Homor sapiens } \\
\text { glucose-6-phosphate dehydrogenase (G6PD), RefSeqGene } \\
\text { (LRG_148) on chromosome X, exon } 9 \\
\text { Origins:Gaozhou, China (Chao, et al., 1991). }\end{array}$ & Germany & nt95 (A>G) Gaohe \\
\hline 4. & $\begin{array}{l}\text { >gi }|122719247| \text { gb | EF190463.1 | Homo sapiens glucose-6- } \\
\text { phosphate dehydrogenase (G6PD) gene, exon } 6 \text { and partial } \\
\text { cds } \\
\text { Origins: Southern China; Mahidol, Thailand (Vulliamy et } \\
\text { al., 1989). }\end{array}$ & China & $\begin{array}{l}\text { nt487(G>A) } \\
\text { Mahidol }\end{array}$ \\
\hline 5. & $\begin{array}{l}\text { >gi }|215769502| \mathrm{dbj}|\mathrm{AB} 376963.1| \text { Homo sapiens G6PD } \\
\text { gene for glucose-6-phosphate dehydrogenase variant, exon } \\
\text { 8, partial cds } \\
\text { Origins: Jammu, India; Gifu, Japan (Beutler et al., 1991). }\end{array}$ & $\begin{array}{l}\text { Papua New Guinea, East } \\
\text { Indonesia, Japan }\end{array}$ & $\begin{array}{l}\text { nt871 (G>A) } \\
\text { Jammu/Viangchan }\end{array}$ \\
\hline 6. & $\begin{array}{l}\text { >gi }|112818958| \mathrm{gb}|\mathrm{DQ} 839546.1| \text { Homo sapiens glucose-6- } \\
\text { phosphate dehydrogenase variant (G6PD) gene, exon } 5 \text { and } \\
\text { partial cds } \\
\text { Origins:Sassari/Cagliari, Italy; Panama, America (Beutler } \\
\text { et al., 1990) }\end{array}$ & $\begin{array}{l}\text { Japan, India, West } \\
\text { Indonesia }\end{array}$ & $\begin{array}{l}\text { nt563 }(\mathrm{C}>\mathrm{T}) \\
\text { Mediterranean }\end{array}$ \\
\hline 7. & $\begin{array}{l}\text { >gi }|111052656| \mathrm{gb}|\mathrm{DQ} 832765.1| \text { Homo sapiens glucose- } \\
\text { 6-phosphate dehydrogenase (G6PD) gene, exons } 3 \text { and } \\
\text { partial cds } \\
\text { Origins: China (Chiu et al., 1993) }\end{array}$ & China & $\begin{array}{l}\text { nt392 }(\mathrm{G}>\mathrm{T}) \\
\text { Chinese- } 4\end{array}$ \\
\hline 8. & $\begin{array}{l}\text { >gi|111052652|gb|DQ832763.1| Homo sapiens glucose- } \\
\text { 6-phosphate dehydrogenase (G6PD) gene, exons } 12 \text { and } \\
\text { partial cds } \\
\text { Origins: Taiwan (Tang et al., 1992) }\end{array}$ & China & $\begin{array}{l}\text { nt493 }(A>G) \\
\text { Chinese-3 }\end{array}$ \\
\hline 9. & $\begin{array}{l}\text { >gi }|111052648| \text { gb } \mid \text { DQ832761.1 | Homo sapiens glucose-6- } \\
\text { phosphate dehydrogenase (G6PD) gene, exon } 7 \text { and partial } \\
\text { cds } \\
\text { Origins:Guangzhou, Southern China (Ren et al., 1999) }\end{array}$ & China & nt175 (C>T) IVS \\
\hline
\end{tabular}




\begin{tabular}{|c|c|c|c|}
\hline No & G6PD and its sequences in NCBI & Distributions & $\begin{array}{c}\text { G6PD mutation } \\
\text { and names }\end{array}$ \\
\hline 10. & $\begin{array}{l}\text { >gi }|111052658| \text { gb } \mid \text { DQ832766.1| Homo sapiens glucose- } \\
\text { 6-phosphate dehydrogenase (G6PD) gene, exons } 4 \text { and } \\
\text { partial cds } \\
\text { Origins:Guangzhou, China; Hakka, Taiwan (Stevens et al., } \\
\text { 1990) }\end{array}$ & China & $\begin{array}{l}\text { nt1376 }(\mathrm{G}>\mathrm{T}) \\
\text { Canton }\end{array}$ \\
\hline 11. & $\begin{array}{l}\text { >gi }|111052654| \mathrm{gb}|\mathrm{DQ} 832764.1| \text { Homo sapiens glucose-6- } \\
\text { phosphate dehydrogenase (G6PD) gene, exon 13, partial } \\
\text { cds } \\
\text { Origins: Guangdong, China; Anand/Dhon India (Chiu et } \\
\text { al., 1991) }\end{array}$ & China & $\begin{array}{l}\text { nt1388 (G>A) } \\
\text { Kaiping }\end{array}$ \\
\hline 12. & $\begin{array}{l}\text { >gi }|111052650| \mathrm{gb}|\mathrm{DQ} 832762.1| \text { Homo sapiens glucose-6- } \\
\text { phosphate dehydrogenase (G6PD) gene, exon } 5 \text { and partial } \\
\text { cds } \\
\text { Origins: Coimbra, Portugal; Shunde, Guangdong, China } \\
\text { (Du et al., 1992) }\end{array}$ & China & $\begin{array}{l}\text { nt592 }(\mathrm{C}>\mathrm{T}) \\
\text { Coimbra }\end{array}$ \\
\hline 13. & $\begin{array}{l}\text { >gi }|31538| \text { emb |X53815.1| Human G6PD gene for } \\
\text { glucose-6-phosphate dehydrogenase, exon 5, partial. } \\
\text { Origins:Shinshu, Japan; Agrigento, Italy (Hirono et al., } \\
\text { 1994a,b) }\end{array}$ & Italy & $\begin{array}{l}\text { nt527 }(A>G) \\
\text { Shinshu }\end{array}$ \\
\hline
\end{tabular}

Table 5. Primers with high identity used to detect G6PDd of Indonesia, China and India

\begin{tabular}{|c|c|c|c|}
\hline Origins & References & \% Identity & G6PD Primers \\
\hline \multirow[t]{2}{*}{ India } & Vulliamy et al., (1991) & $71.3 \%$ & nt1376-F (5'-ACGTGAAGCTCCTGACGC-3') \\
\hline & & $82.2 \%$ & nt1388-R (5'-GTGCAGCAGTGGGGTGAACATA-3') \\
\hline \multirow[t]{3}{*}{ Indonesia } & Matsuoka et al., (2003) & $94 \%$ & nt1388-F (5'-TGAGGGTGGAGCTACCTCAT-3') \\
\hline & Kawamoto et al., (2006) & $96.9 \%$ & nt1376-R (5'-CACCATGTGGAGTCCCCCGG-3') \\
\hline & $\begin{array}{l}\text { Soemantri et al., (1995) } \\
\text { Tantular et al., (2010) }\end{array}$ & & \\
\hline \multirow[t]{2}{*}{ China } & Matsuoka et al., (2007) & $70.9 \%$ & nt163-F (5'-GGAGCTAAGGCGAGCTCTGGC-3') \\
\hline & & $70.7 \%$ & nt1311-R (5'-AAGACGTCCAGGATGAGGTGATC-3') \\
\hline \multirow{2}{*}{$\begin{array}{l}\text { Indonesia, } \\
\text { India, China }\end{array}$} & Matsuoka et al., (2007) & $58.7 \%$ & nt1376-F (5'-CTGAGAGAGCTGGTGCT-3') \\
\hline & & $63.9 \%$ & nt1311-R (5'-ATGAGGTAGCTCCACCCTCA-3') \\
\hline
\end{tabular}

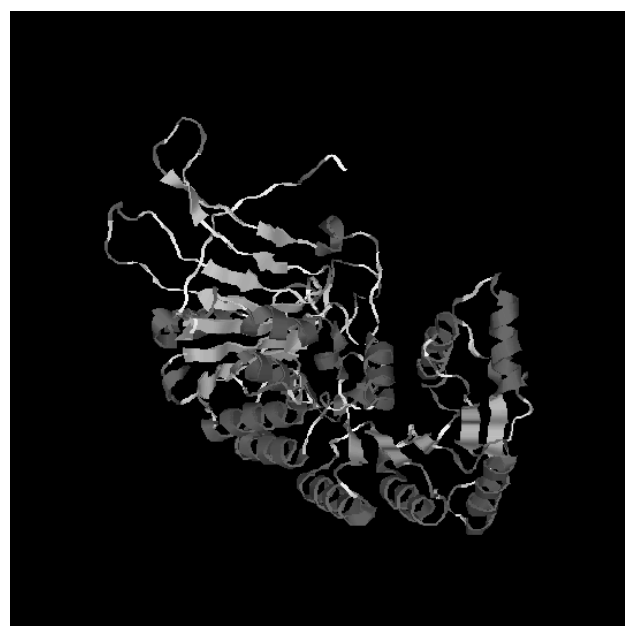

Figure 1. Predicted G6PD protein structure (3.6 $\pm 2.5 \AA ̊$ RMSD from i-TASSER Michigan University) 
Table 6. Haplotypes of G6PD genes in different polymorphic sites (Vulliamy et al., 1991)

\begin{tabular}{lccc}
\hline Characteristics & G6PD genes & G6PD genes & G6PD gene \\
\hline Base change & nt175 C>T & nt163 C>T & nt1311 C > T \\
Exon number & 7 & 8 & 11 \\
Restriction sites & ScaI & BspHI & BclI \\
Distributions & Chinese and Indian & Chinese and Indian & $\begin{array}{c}\text { Iranian/European, Javanese-Indonesian } \\
\text { and Indian }\end{array}$ \\
Haplotypes & A and A- & B and A & B \\
\hline
\end{tabular}

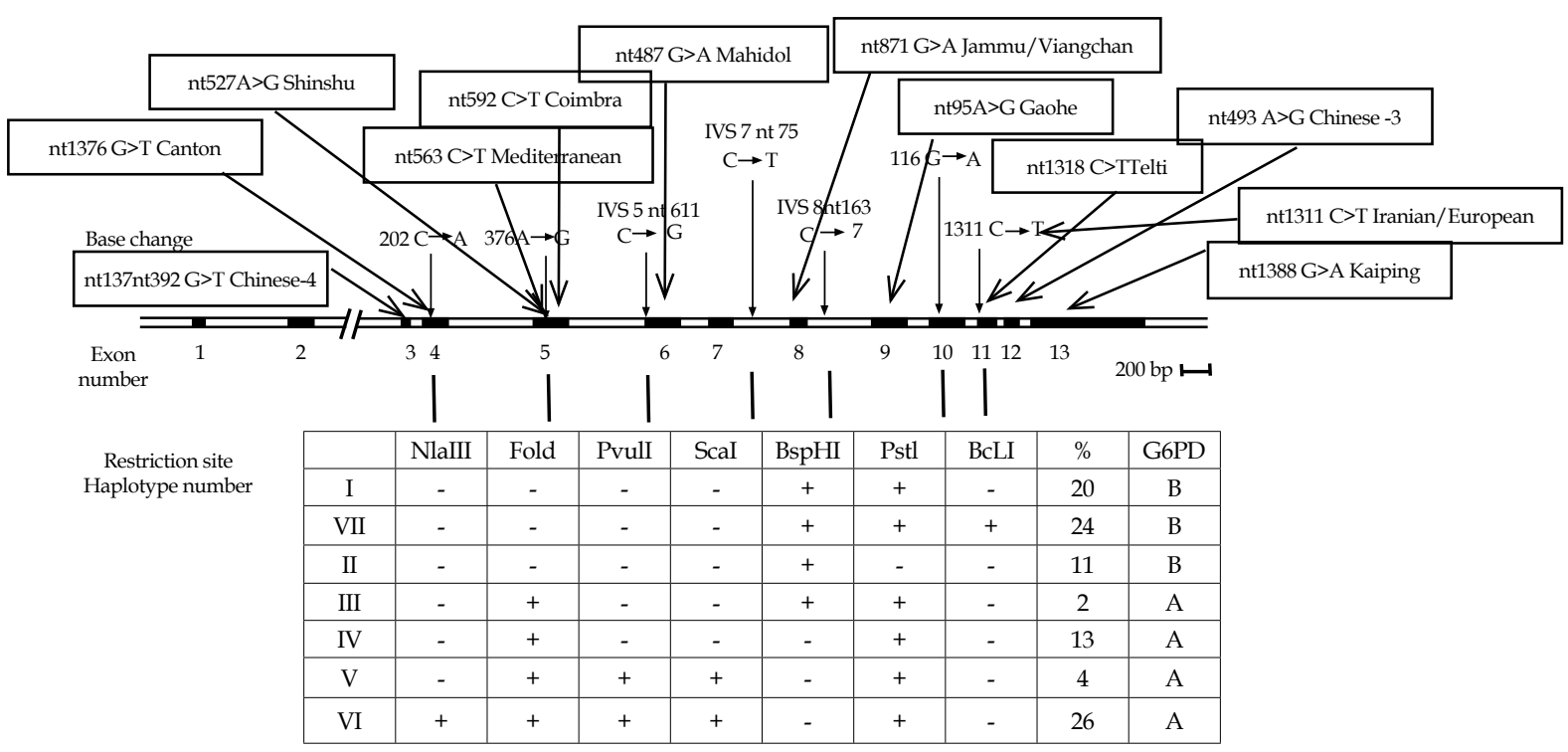

Figure 2. Haplotypes of G6PD and locations of polymorphic sites (Vulliamy et al., 1991)

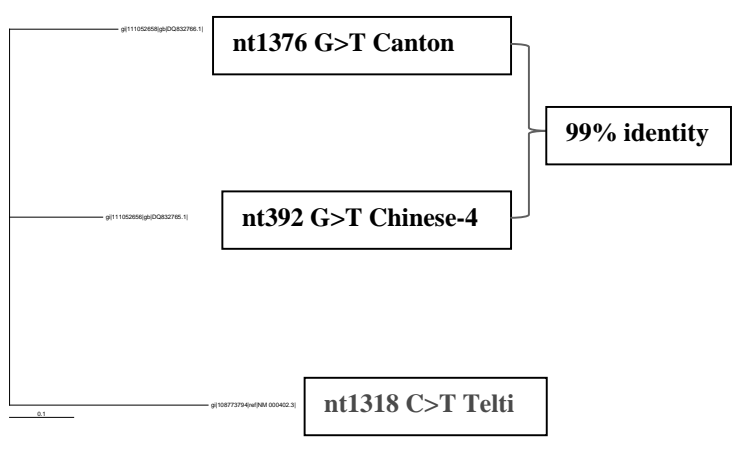

Figure 3. nt392 G>T Chinese-4 and nt1376 G>T Canton (99\% high identity) bounded by Chinese primers of nt1376-F (5'-CTGAGAGAGCTGGTGCT-3') and nt1311-R (5'-ATGAGGTAGCTCCACCCTCA-3') with the outgroup of nt1318 C>T Telti. The PCR product is $90 \mathrm{bp}$ and the block of aligned sequence is $910 \mathrm{bp}$.

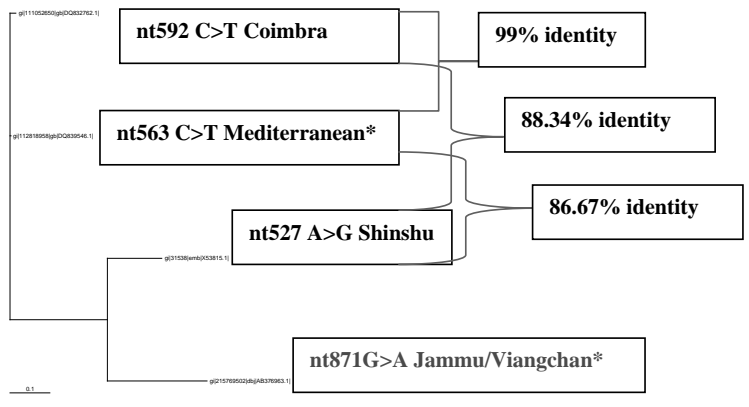

Figure 4. nt563 C>T Mediterranean and nt592 C>T Coimbra (99\% high identity), nt527 A $>$ G Shinshu and nt563 C>T Mediterranean (86.67\% moderate identity), nt527 A>G Shinshu and nt592 C>T Coimbra $(88.34 \%$ moderate identity), bounded by Chinese primers of nt1376-F (5'-CTGAGAGAGCTGGTGCT-3') and nt1311-R (5'-ATGAGGTAGCTCCACCCTCA-3') with the outgroup of nt871 G>A Jammu/Viangchan. The PCR product is $60 \mathrm{bp}$ and the block of aligned sequence is 580 bp.Asterisk (*) is G6PD mutation located in Indonesia 
Table 7. G6PD mutated genes and regions inchromosome (Xq28)(http://www.ncbi.nlm.nih.gov/)

\begin{tabular}{|c|c|c|c|}
\hline Regions & G6PD mutated genes & NCBI & $\%$ Identity \\
\hline Region 3 & nt392 & >gi|111052656|gb|DQ832765.1| & $99 \%$ between a 9 \\
\hline Region 4 & $\begin{array}{l}\text { nt1376 G>T Canton* } \\
\text { (Figure 3) }\end{array}$ & >gi|111052658 | gb|DQ832766.1 & Chinese -4 and Canton $=\mathrm{H}$ \\
\hline Region 5 & $\begin{array}{l}\text { nt527 A }>\text { G Shinshu } \\
\text { nt563 C>T } \\
\text { Mediterranean* } \\
\text { nt592 C > T Coimbra } \\
\text { (Figure 4) }\end{array}$ & $\begin{array}{l}\text { >gi |31538 | emb |X53815.1| } \\
\text { >gi | } 112818958|\mathrm{gb}| \mathrm{DQ} 839546.1 \mid \\
\text { >gi|111052650|gb|DQ832762.1| }\end{array}$ & $\begin{array}{l}\text { - } 99 \% \text { between a } 580 \mathrm{bp} \\
\text { region enclosing Coimbra } \\
\text { andMediterranean = High identity } \\
\text { - } 88.34 \% \text { between a } 580 \text { bp region } \\
\text { enclosingShinshu and Coimbra = } \\
\text { Moderate identity } \\
\text { - } 86.67 \% \text { between a } 580 \mathrm{bp} \\
\text { region enclosingShinshu and } \\
\text { Mediterranean = Moderate identity }\end{array}$ \\
\hline & nt487 G> & $>$ gi $\mid 122$ & $74.07 \% \mathrm{k}$ \\
\hline Regi & $\begin{array}{l}\text { nt175 C > T IVS } \\
\text { (Figure 5) }\end{array}$ & >gi|111052648|gb|DQ832761.1| & $\begin{array}{l}\text { enclosing Mahidol and IVS = Low } \\
\text { identity }\end{array}$ \\
\hline Region 8 & nt871 G>A Jammu/ & >gi|215769502|dbj|A & $92.5 \%$ similarity between a $720 \mathrm{bp}$ \\
\hline Region 9 & $\begin{array}{l}\text { Viangchan* } \\
\text { nt95 A }>\text { G Gaohe } \\
\text { (Figure 6) }\end{array}$ & 385264 | ref | NG_009015.1| & $\begin{array}{l}\text { region enclosing Jammu/Viangchan } \\
\text { and Gaohe= Moderate identity }\end{array}$ \\
\hline Region 11 & $\begin{array}{l}\text { nt1318 C>T Telti } \\
\text { nt1311 C>T Iranian/ } \\
\text { European } \\
\text { (Figure 7) }\end{array}$ & $\begin{array}{l}\text { >gi } \mid 108773792 \text { |ref |NM_0010423 } \\
\text { 51.1| } \\
\text { >gi }|108773794| \text { ref |NM_000402.3| }\end{array}$ & $\begin{array}{l}99 \% \text { similarity between a } 550 \text { bp region } \\
\text { enclosing Telti and Iranian/European = } \\
\text { High identity }\end{array}$ \\
\hline Region 12 & nt493 A>G Chinese -3 & >gi|111052652|gb|DQ832763.1| & $74.12 \%$ betweena 830 bp reg \\
\hline Region 13 & $\begin{array}{l}\text { nt1388 G>A Kaiping* } \\
\text { (Figure 8) }\end{array}$ & >gi|111052654|gb|DQ832764.1| & $\begin{array}{l}\text { enclosing Chinese- } 3 \text { and Kaiping= Low } \\
\text { identity }\end{array}$ \\
\hline
\end{tabular}

Note: * (asterisk) indicates the mutated G6PD genes located in Indonesia

Phylogenetic tree (Figure 4) was generated based on a block of $580 \mathrm{bp}$ that includes mutations at 527 , at 563 and at 592 in adjacent region 5 of G6DP on chromosome Xq28 (Table 8).

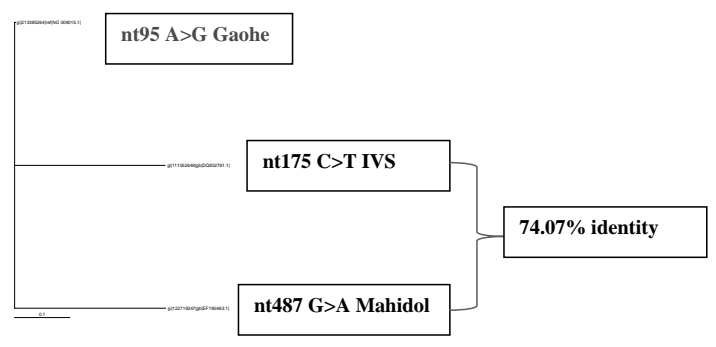

Figure 5. nt487 G>A Mahidol and nt175 C>T IVS (74.07\% low identity), bounded by Chinese primers of nt1376-F (5'-CTGAGAGAGCTGGTGCT-3') and nt1311-R (5'-ATGAGGTAGCTCCACCCTCA-3') with the outgroup of nt95 A>G Gaohe. The PCR product is $120 \mathrm{bp}$ and the block of aligned sequence is $520 \mathrm{bp}$.
Phylogenetic tree (Figure 5) was generated based on a block of $520 \mathrm{bp}$ that includes mutations at 175 and at 487 in adjacent regions 6 and 7 of G6DP on chromosome Xq28 (Table 8).

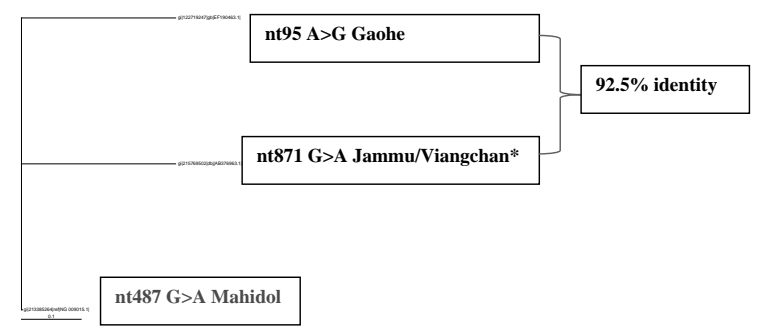

Figure 6. $n t 871 \mathrm{G}>\mathrm{A}$ Jammu/Viangchan and $\mathrm{nt} 95 \mathrm{~A}>\mathrm{G}$ Gaohe (92.5\% moderate identity), bounded by Chinese primers of nt1376-F (5'-CTGAGAGAGCTGGTGCT-3') and nt1311-R (5'-ATGAGGTAGCTCCACCCTCA-3') with the outgroup of nt 487 G>A Mahidol. The PCR product is $80 \mathrm{bp}$ and the block of aligned sequence is 720 bp. Asterisk (*) is G6PD mutation located in Indonesia. 


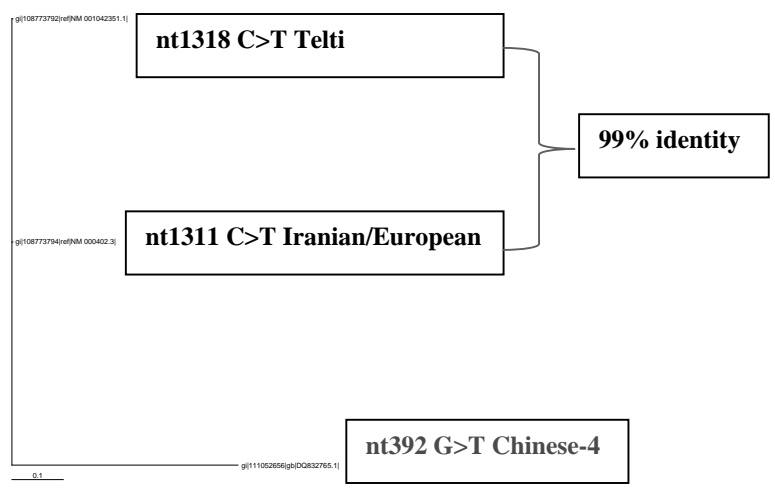

Figure 7. nt1318 C>T Telti and nt1311 C>T Iranian/ European (99\% high identity), bounded by Chinese primers of nt1376-F (5'-CTGAGAGAGCTGGTGCT-3') and nt1311-R (5'-ATGAGGTAGCTCCACCCTCA-3') with the outgroup of nt392 G>T Chinese-4. The PCR product is $90 \mathrm{bp}$ and the block of aligned sequence is $550 \mathrm{bp}$.

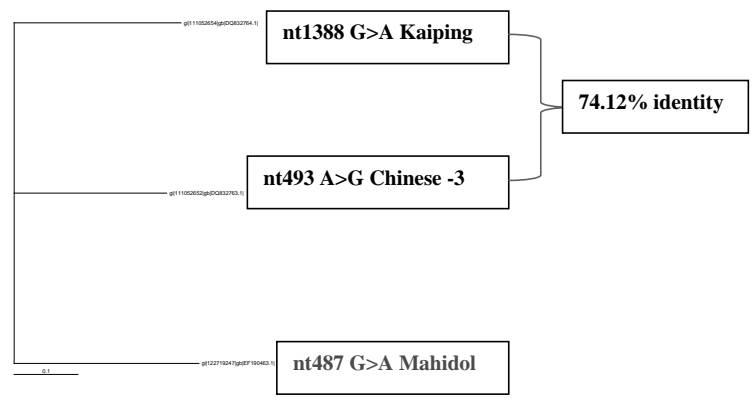

Figure 8. nt493 A>G Chinese -3 and nt1388 G>A Kaiping (74.12\% low identity), bounded by Chinese primers of nt1376-F (5'-CTGAGAGAGCTGGTGCT-3') and nt1311-R (5'-ATGAGGTAGCTCCACCCTCA-3') with the outgroup of $n t 487 \mathrm{G}>\mathrm{A}$ Mahidol. The PCR product is $190 \mathrm{bp}$ and the block of aligned sequence is $830 \mathrm{bp}$.Asterisk (*) is G6PD mutation originally located in Indonesia.

Phylogenetic tree (Figure 6) was generated based on a block of $720 \mathrm{bp}$ that includes mutations at 95 and at 871 in adjacent regions 8 and 9 of G6DP on chromosome Xq28 (Table 7).

Phylogenetic tree (Figure 7) was generated based on a block of $550 \mathrm{bp}$ that includes mutations at 1311 and at 1318 in adjacent region 11 of G6DP on chromosome Xq28 (Table 7).

Phylogenetic tree (Figure 8) was generated based on a block of $830 \mathrm{bp}$ that includes mutations at 493 and at 1388 in adjacent regions 12 and 13 of G6DP on chromosome Xq28 (Table 7).

\section{Discussion}

The predicted protein structure of G6PD mutation showed a monomer protein with a substrate binding cavity between two domains (Figure 1). The locations of amino acid mutations are at the binding sites, presumed to have functions in protection against malaria (Mason, et al., 2007). The polymorphic mutations decrease stability of the enzyme in red cells, by disturbing protein folding, affecting amino acid residues at dimer interface or interacting with a structural NADP molecule that stabilizes the enzyme (Mason, et al., 2010).As discussed by Turner et al. (2001), a number of different G6PD variants have reached polymorphic frequencies in different parts of the world due to the relative protection they confer against malaria infection. Mason et al. (2007) showed that very rarely de novo mutations can arise causing the more severe condition of chronic nonspherocytic hemolytic anemia. Altogether 169 different mutations were described (Mason et al., 2010). Iwai et al. (2001) showed that G6PD mutations in Chinese are heterogeneous and at least 10 mutations have been reported including G6PD Canton and G6PD Kaiping. Kaiping mutations specifically showed minimal mobility shift. Interestingly, Iwai et al. (2001) Soemantri et al. (1995) also found that G6PD Kaiping was not found in orang asli (original people) from Indonesia, India, Malaysia and Myanmar, but was found in Chinese, Singaporean Chinese and Taiwan Chinese. G6PD Canton was found in Javanese Indonesian, Chinese, Singaporean Chinese and Taiwan Chinese. However, further study by Matsuoka et al. (2003) revealed that G6PD Kaiping was indeed found in Maumere and Telibura at Flores Island, Indonesia while G6PD Canton was found in Central Java, Indonesia. It is important to note that the history of Flores Island, in East Indonesia, is consistent with a diverse origin of mutations. 
It belongs to the Sunda archipelago where people come from Eurasian countries, African countries, Philippine Islands, or Pacific islands. Therefore, historically, Flores Islands might have received many tribes from different origins. The high frequency distribution of G6PD Kaiping, a common variant in Chinese population, in the Sikka, Flores Islands is a rather unexpected finding (Kawamoto et al., 2006). In contrast, Jiang et al. (2006) characterized that G6PD Canton was never seen in the Sikka, Flores Islands nor in the Ende, Timor Islands, although many studies revealed that G6PD Canton in China, Taiwan, Thailand, Malaysia, and Singapore is found at higher than or almost equal in frequency to G6PD Kaiping in those countries. Bioinformatics is needed to discover the genetic relationships between mutations that are common in Indonesia, and those found in China, India and the Middle East. For instance, mutations at nt175, nt163 and nt1311 are summarized in Table 6. Haplotype number V and VI are thought to be from Chinese origins whereas haplotype number VII are from Javanese Indonesians and Indians, as studied by Vulliamy et al. (1991). Additionally, it was presumed by Kawamoto et al. (2006) that Chinese traders or immigrants have shared genes with Christian Sikka women and so introduced G6PD Kaiping mutated gene into the Sikka population in Flores Islands, Indonesia. However, Indonesian G6PD mutated genes also consist of G6PD Jammu/Viangchan in East Indonesia (including Flores Islands). It is clear from Soemantri et al. (1995) that G6PD deficiency is found in Java Island, Indonesia and is represented by the G6PD mutations of Mediterranean and G6PD mutations of Canton, consistent with a Mediterranean, Indian and Chinese ("Mongoloid" is the term used by Soemantri et al. (1995) contribution to the gene pool of the Javanese. G6PD Mediterranean in West Indonesia (including Java Island) is most closely related to Chinese G6PD mutated genes (G6PD Coimbra) with 99\% identity (see Figure 4). From Table 6 and
Figure 2, it can be seen that Flores Indonesian G6PD deficiency (Jammu/Viangchan) is moderately related ( $92.5 \%$ identity) to Chinese G6PD mutation (Gaohe) (see Table 7 and Figure 6). The phylogenetic tree of regions containing mutation bounded by Chinese PCR primers is shown in Figure 3, 4, 5, 6, 7 and 8 . The block containing the G6PD mutations found in Indonesia(nt563 C>T Mediterranean) is highly related to Chinese G6PD (nt592 C>T Coimbra) with 99\%identity between the regions containing these gene mutations(see Figure 4). Indonesian G6PD (Mediterranean) and Chinese G6PD (Coimbra) are located in the same exons (at exon 5) as well as possessing high identity in the sequence adjacent to the mutations. The G6PD mutation found in Indonesia (nt563 C $>$ T Mediterranean) is moderately related to Chinese G6PD (which is also found in Italy, nt527 A>G Shinshu) with 86.67\% identity (Figure 4). Both are located in exon 5. Chinese G6PD mutation (Shinshu)is moderately related to Chinese (Coimbra) G6PD mutation with $88.34 \%$ identity (see Figure 4 ). A phylogenetic tree of regions containing G6PD segments bounded by Chinese PCR primers showed $92.5 \%$ (moderate) identity (Figure 6) between G6PD found in Indonesia (nt871 G>A Jammu/Viangchan) and Chinese G6PD(nt95 A>G Gaohe). It can be concluded from the phylogenetic trees that G6PD Mediterranean in Indonesia is most closely related to G6PD Coimbra, while G6PD Jammu/Viangchan in Indonesia has less similarity with G6PDGaohe. The block of DNA bounding G6PD Mahidol is more distantly related to G6PD IVS with $74.07 \%$ (low identity)(see Figure 5), whereas G6PD Iranian/European has high identity (99\%) with G6PD Telti, found in India (see Figure 7). It is important to note that G6PD Canton, found in Java Island, West Indonesia, is closely related to G6PD Chinese-4 with 99\% high identity (see Figure 3). Meanwhile G6PD Kaiping, mostly distributed in Flores Island, East Indonesia is more distantly related to G6PD Chinese-3 with $74.12 \%$ identity (see 
Figure 8). G6PD Kaiping is found not only in Flores Island but also in Java Island. G6PD Jammu/Viangchan is only found in Flores Island. In addition, there were other phylogenetic trees constructed for reviewing the best primers with high percentages of identity for amplifying samples from various locations. The primers with high percentages of identity for use with Indonesian samples are the Chinese primers (i.e. a pair of primers of nt1388-F and nt1376-R), and other Chinese primers of nt1376-F and nt1311-R (for Indonesian, Indian and Chinese samples). The Chinese primers of nt1376-F and nt1311-R for Indonesian, Indian and Chinese generate products that fall within the blocks of DNA used to generate phylogenetic trees for regions containing the mutations of the G6PD genes. The primers can be used to detect G6PD mutated gene samples, as summarized in Table 5. Based on our phylogenetic trees and the percentages of identity between blocks of DNA sequence enclosing the mutations (see Table 7), we conclude that the Flores Indonesian G6PD deficiency (Jammu/ Viangchan G6PD), which is located in the eastern parts of Indonesia is moderately $(92.5 \%)$ related to the G6PD deficiency (GaoheG6PD) of Chinese origin, consistent with a distant Chinese origin for this mutation, but with significant evolutionary divergence between these regions, which is likely to have occurred prior to introduction of the Jammu/ Viangchan mutation into Indonesia. It was interesting to note that in the region containing the PCR product bounded by PCR primer pair of nt1376 and nt1311, theJavanese Indonesian G6PD deficiency (Mediterranean G6PD)is highly related (99\%) to the Chinese G6PD deficiency (Coimbra G6PD), but only moderately related to Shinshu G6PD deficiency. It has been suggested that the originally reported G6PD mutations in Java Island (Jakarta, Solo and Surabaya) and Bali Island (Coimbra G6PD, Shinshu G6PDand Canton G6PD); as well as G6PD mutation is Flores Island and east Indonesian islands (Jammu/Viangchan G6PD, Gaohe G6PD and
Kaiping G6PD) were introduced by Chinese migrants since the year 1293, whilst G6PD mutations in Sumatra Island (Medan) and Java Island (Jakarta and Bandung) (Mediterranean G6PD) were introduced byIndian traders since the first century (Brandes, 1913; Tantular, et al., 2010). However, our results support the suggestion that a mutation in Java and west Indonesia (Mediterranean G6PD) is very closely related to Chinese G6PD deficiency (Coimbra G6PD), whilst the mutation originating in India (Jammu/Viangchan G6PD) and now distributed in east Indonesia, is moredistantly related to Gaohe G6PD deficiency of Chinese origin.

\section{Acknowledgments}

This work was funded by Australian Leadership Award from Ausaid. Thank you to Ari Satyagraha for help during this work.

\section{References}

Beutler, E., and Kuhl, W. 1990. The NT 1311 polymorphism of G6PD: G6PD Mediterranean mutation may have originated independently in Europe and Asia. Am. J. Hum. Genet.,47, 1008-1012.

Beutler, E., Prchal, J.T., Westwood, B., and Kuhl, W.1991. Definition of the mutations of G6PD Wayne, G6PD Viangchan, G6PD Jammu and G6PD "LeJeune". Acta Haematol., 86, 179-182.

B r a n d e s, J. L. A . ( 1913 ). O u d $\mathrm{J}$ a v a a s c h e o o r k o d e n : nagelatentransscripties van wijlen J.L.A. Brandes ; uitgegeven door N.J. Krom. Batavia: Albrecht.

Cappellini, M.D., and Fiorelli, G., 2008. Glucose-6-phosphate dehydrogenase deficiency. Lancet, 371(9606), 64-74.

Chao, L., Du, C.S., and Louie, E. 1991. A to $\mathrm{G}$ substitution identified in exon 2 of the G6PD gene among G6PD deficient Chinese. Nucleic Acids Res., 19, 6056.

Chiu, D.T.Y., Zuo, L., and Chao, L. 1993. Molecular characterization of glucose- 
6-phosphate dehydrogenase (G6PD) deficiency in patients of Chinese descent and identification of new base substitutions in the human G6PD gene. Blood,81, 2150-2154.

Chiu, D.T.Y., Zuo, L., and Chen, E. 1991. Two commonly occurring nucleotide base substitutions in Chinese G6PD variants. Biochem.Biophys. Res.Commun., 180,988993.

Clustal-X., 2012. Clustal-X v 2.1.Available from http://www.clustal.org/ on 21 July 2012.

Du, C.S., Chao, L.T., Louie, E., Liu, T.Z., and Chiu, D.T.Y. 1992.Molecular characterization of G6PD deficiency in patients of Chinese descent and identification of a new base substitution in the human G6PD gene.Blood, 80 (Suppl 1), 284a.

Frank, J. E., 2005. Diagnosis and management of G6PD deficiency. Am. Fam. Physician., 72(7), 1277-82.

Hamilton, J. W., Jones, F. G., and McMullin, M. F.,2004.G6PDGuadalajara: acase of chronic non-spherocytichaemolyticanaemia responding to splenectomy and the role of splenectomy in this disorder. Hematology, 9(4), 307-9.

Hirono, A., Miwa, S., Fujii, H., Ishida, F., Yamada, K., Kubota, K. 1994a.Molecular study of eight Japanese cases of G6PD by nonradio isotopic single-strand conformation polymorphism analysis. Blood,83,3363-3368.

Hirono, A., Nakayama, S., Fujii, H., and Miwa, S. 1994b. Molecular abnormality of a unique Japanese G6PD variant (G6PD Kobe) with a greatly increased affinity for galactose-6-phosphate. Am. J. Hematol.,45, 185-186.

i-TASSER., 2012. i-TASSER results for job id S110585. Available from http:/ / zhanglab. ccmb.med.umich.edu/I-TASSER/ output/S110585 on 21 August 2012.

Iwai, K., Hirono, A., Matsuoka, H., Kawamoto, F., Horie, T., Lin, K., Tantular, I. S., Dachlan, Y. P., Notopuro, H., Hidayah,
N. I., Salim, A. M. A., Fujii, H., Miwa, S., and Ishii, A., 2001. Distribution of glucose-6-phosphate dehydrogenase mutations in Southeast Asia. Hum. Genet., 108, 445-449.

Jiang, W., Yu, G., Liu, P., Geng, Q., Chen, L., Lin Q., Ren, X., Ye, W., He, Y., Guo Y., Duan, S., Wen, J., Li, H., Qi, Y., Jiang, C., Zheng, Y., Liu, C., Si, E., Zhang, Q., Tian, Q., and Du, C., 2006. Structure and function of glucose-6-phosphate dehydrogenasedeficient variants in Chinese population. Hum. Genet., 119, 463-478.

Kawamoto, F., Matsuoka, H., Kanbe, T., Tantular, I.S., Pusarawati, S., Kerong, H. I., Damianus, W., Mere, D., and Dachlan, Y. P., 2006. Further investigation of glucose6-phosphate dehydrogenase variants in Flores Island, eastern Indonesia. J. Hum. Genet., 51, 952-957.

Mason, P. J., Bautista, J. M., and Gilsanz, F., 2007. G6PD deficiency: the genotypephenotype association. Blood Rev., 21(5), 267-283.

Mason, P. J., Bautista, J. M., and Gilsanz, F., 2010. Corrigendum to G6PD deficiency: the genotype-phenotype association. Blood Rev., 24(1), 49.

Mason, P.J., Sonati, M.F., and MacDonald, D. 1995. New glucose-6-phosphate dehydrogenase mutations associated with chronic anemia. Blood. 85, 13771380.

Matsuoka, H., Arai, M., Yoshida, S., Tantular, I. S., Pusarawati, S., Kerong, H., and Kawamoto, F., 2003. Fivedifferent glucose6-phosphate dehydrogenase (G6PD) variants found among 11 G6PD-deficient persons in Flores Island, Indonesia. $J$. Hum. Genet., 48, 541-544.

Matsuoka, H., Thuan, D. T. V., Thien, H. V., Kanbe, T., Jalloh, A., Hirai, M., Arai, M., Dung, N., T., and Kawamoto, F., 2007. Seven different glucose-6-phosphate dehydrogenase variants including a new variant distributed in Lam Dong province in Southern Vietnam. Acta. Med. Okayama., 61(4), 213-219. 
Mazza, J. (2001). Manual of clinical hematology. New York: Lippincott Williams and Wilkins.

McKusick, V, A., and Hamosh, A., 2010. Anemia, nonspherocytic hemolytic, due to G6PD deficiency.Available from http:/ / omim.org/entry/305900

Mehta, A., Mason, P. J., and Vulliamy, T. J., 2000. Glucose-6-phosphate dehydrogenase deficiency.Baillieres Best Pract. Res. Clin. Haematol.,13(1), 21-38.

Monga, A., Makkar, R.P., Arora, A., Mukhopadhyay, S., and Gupta, A.K., 2003. Case report: Acute hepatitis E infection with coexistent glucose-6phosphate dehydrogenase deficiency. Can. J. Infect. Dis.,14(4), 230-1.

Mortazavi, Y.,Chopra, R., Gordon-Smith, E.C., and Rutherford, T.R. 1997. Frequency of the G6PD nt $1311 \mathrm{C} / \mathrm{T}$ polymorphism in English and Iranian populations: relevance to studies of $\mathrm{X}$ chromosome inactivation. J Med Genet., 34(12), 1028-9.

NCBI., 2012. G6PD and Homo sapiens. Available from http://www.ncbi.nlm. nih.gov/nuccore

Ren, X.,Du, C., and Lin, Q. 1999. Studies on a G6PD polymorphic site, C1311T. ZhonghuaXue Ye XueZaZhi, 20(4), 197-9.

Satyagraha, A. W. (2012). G6PD Testing in Indonesia. Incheon: Asia Pacific Malaria Elimination Network (APMEN).

Soemantri, A. G., Saha, S., Saha, N., and Tay, J. S. H., 1995. Molecular variants of red cell glucose-6-phosphate dehydrogenase deficiency in Central Java, Indonesia. Hum. Hered., 45, 346-350.

Stevens, D.J., Wanachiwanawin, W., Mason, P.J., Vulliamy, T.J., and Luzzatto, L. 1990. G6PD Canton a common deficient variant in South East Asia caused by a 459 Arg->Leu mutation. Nucleic Acids Res, 18, 7190.

Tang, T.K., Huang, C.S., Huang, M.J., Tam, K.B., Yeh, C.H., and Tang, C.J.C. 1992. Diverse point mutations result in glucose 6-phosphate dehydrogenase (G6PD) polymorphism in Taiwan. Blood, 79, 2135-2140.

Tantular, I. S., Matsuoka, H., Kasahara, Y., Pusarawati, S., Kanbe, T., Tuda, J. S. B., Kido, Y., Dachlan, Y. P., and Kawamoto, F., 2010. Incidence and mutation analysis of glucose-6-phosphate dehydrogenase deficiency in Eastern Indonesian populations.Acta. Med. Okayama., 64(6), 367-373.

Turner, P.C., McLennan, A.G., Bates, A.D., and White, M. R. H. (2001). Molecular Biology. Second edition. New Delhi: BIOS Scientific Publishers Limited.

Vulliamy, T. J., Othman, A., Town, M., Nathwani, A., Falusi, A. G., Mason, P. J., and Luzatto, L., 1991. Polymorphic sites in the African population detected by sequence analysis of the glucose-6phosphate dehydrogenase gene outline the evolution of the variants A and A-. Proc. Nat. Acad. Sci., 88, 8568-8571.

Vulliamy, T.J., Wanachiwanawin, W., Mason, .PJ.,and Luzzatto, L. 1989. G6PD Mahidol, a common deficient variant in South East Asia is caused by a (163)glycine-serine mutation. Nucleic Acids Res., 17, 5868.

Warrel, D. A., Cox, T. M., Firth, J. D., and Benz, E. J. (2005). Oxford: Oxford University Press. 